\title{
Le révolutionnaire qui alluma la mèche : Ahmed Niyazi Bey de Resne
}

Niyazi Bey from Resne: the Revolutionary who lit the Fuse

Fitili Ateşleyen İhtilâlci : Resne'li Niyazi Bey

Faruk Bilici

\section{OpenEdition}

\section{Journals}

Édition électronique

URL : https://journals.openedition.org/ceb/1052

DOI : 10.4000/ceb.1052

ISSN : 2261-4184

Éditeur

INALCO

Édition imprimée

Date de publication : 9 janvier 2012

ISSN : 0290-7402

\section{Référence électronique}

Faruk Bilici, «Le révolutionnaire qui alluma la mèche : Ahmed Niyazi Bey de Resne », Cahiers

balkaniques [En ligne], 40 | 2012, mis en ligne le 31 mars 2012, consulté le 06 juillet 2021. URL : http:// journals.openedition.org/ceb/1052 ; DOI : https://doi.org/10.4000/ceb.1052

Ce document a été généré automatiquement le 6 juillet 2021.

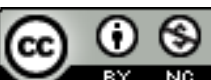

Cahiers balkaniques est mis à disposition selon les termes de la Licence Creative Commons Attribution - Pas d'Utilisation Commerciale 4.0 International. 


\title{
Le révolutionnaire qui alluma la mèche : Ahmed Niyazi Bey de Resne
}

\author{
Niyazi Bey from Resne: the Revolutionary who lit the Fuse \\ Fitili Ateşleyen İhtilâlci : Resne'li Niyazi Bey
}

\section{Faruk Bilici}

1 Si la Révolution jeune-turque a été préparée et alimentée par des élites dans les grandes villes ottomanes (Istanbul, Salonique, Izmir), mais aussi par des intellectuels opposants, réfugiés à Paris, à Genève ou encore au Caire, sans l'implication directe et sur le terrain de l'armée, elle n'aurait probablement pas pu avoir lieu ${ }^{1}$. Or, le rôle de la Troisième armée basée en Macédoine, et plus particulièrement celui d'un certain nombre de jeunes officiers, fut déterminant. En effet, l'armée ottomane est le lieu par excellence de la mise en application des «utopies" positivistes des officiers formés dans les meilleures écoles de l'empire. C'est une formation basée, surtout à partir des années 1880 , sur de nouvelles solidarités impersonnelles visant à créer, non pas une allégeance à la dynastie ottomane, mais un nouveau type de citoyen voué à "sauver la patrie ». Formés par les meilleurs enseignants, dont nombre sont d'origine étrangère, parlant des langues occidentales, en contact avec la littérature et les sciences européennes, les cadets ottomans d'extraction souvent populaire méprisent toute la vieille garde et le moule hiérarchique fondé sur la relation maître / serviteur. Devant l'effritement de l'empire engendré par chaque guerre, les dépenses excessives d'argent emprunté à grands frais aux organismes financiers occidentaux, la corruption généralisée autour du palais, l'impuissance politique pour répondre aux aspirations politiques des couches sociales et ethniques, l'officier ottoman éprouve naturellement des sentiments de contestation.

2 L'un d'entre eux, Halil Pacha, résume l'état d'esprit de cette génération d'officiers :

«La gestion imbécile du palais nous créait tous les jours de nouvelles catastrophes. Si nous ne donnions pas une nouvelle forme à la direction de l'État, ce désordre pouvait continuer encore des années [...] Cela n'avait pas de fin et l'État se perdait. Si nous, nous ne tendions pas la main à la patrie avec intelligence, qui pouvait le faire [...] ? L'idée de faire disparaître 
l'influence du palais, et la remplacer par la main puissante du peuple était ancrée dans nos têtes depuis les bancs de l'école "².

\section{Niyazi : de la Guerre turco-grecque à la Révolution jeune-turque}

C'est à la biographie de l'un de ces officiers ottomans, "prêts à faire disparaître l'influence d'Abdülhamid et à la remplacer par la main du peuple » que cette étude est consacrée.

\section{Niyazi Bey}

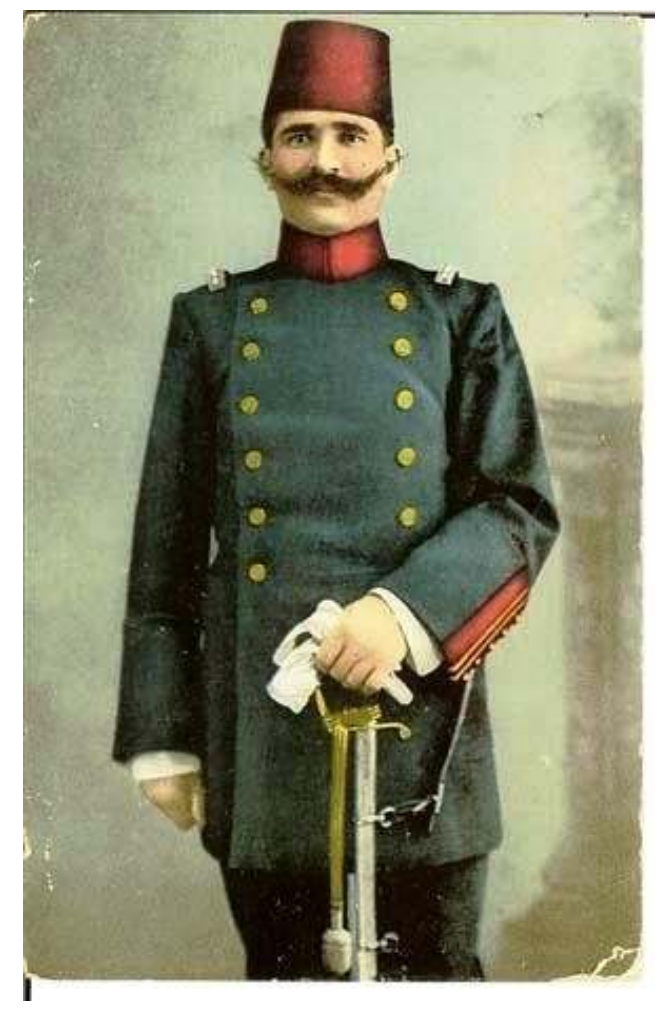

4 Après le traité de Berlin, près de la moitié de l'armée ottomane est mobilisée pour la défense des vilayets de la Macédoine, la totalité de la Troisième armée (basée parfois à Salonique, parfois à Monastir), fondée en 1843, une partie de la Deuxième armée (basée à Edirne) ainsi que différentes unités prélevées sur les autres armées, affaiblissant ainsi dangereusement la défense des régions anatoliennes et arabes. Cette Troisième armée, chargée de protéger les provinces balkaniques contre les nouveaux États hostiles à l'Empire ottoman, était également chargée d'assurer la sécurité à l'intérieur des territoires appartenant à l'empire, malgré la présence de la gendarmerie, de la police et des gardes champêtres. De plus, les soldats recrutés pour cette Troisième armée étaient essentiellement originaires des Balkans, autrement dit, ils vivaient quotidiennement les conflits régionaux, voire familiaux, avec les populations chrétiennes locales.

5 Par ailleurs, les meilleurs officiers, diplômés de l'Académie militaire, de l'École de guerre ou des écoles militaires allemandes étaient affectés à la Troisième armée. Instruits et ouverts aux idées libérales, ces officiers allaient poser le problème des 
inégalités, des dysfonctionnements dans l'armée, des problèmes d'identité nationale des populations non-musulmanes contre lesquelles ils devaient lutter.

Devenu chronique depuis la guerre de Crimée (1853-1856), le retard, ou même le nonversement, des salaires des officiers de l'armée et de la gendarmerie, est le premier grief que soldats et officiers expriment à travers les manifestations et les mutineries tout au long du dernier quart du XIX ${ }^{e}$ siècle. Cette injustice est devenue encore plus flagrante à partir de 1903, lorsque la Macédoine est divisée en cinq zones de sécurité où les contingents de gendarmes étrangers devaient travailler avec les gendarmes ottomans dont une bonne partie venait de l'armée régulière. Considérée comme une intervention détournée, cette présence étrangère était surtout perçue comme un soutien aux populations chrétiennes exercé au détriment des populations musulmanes. De plus, les écarts de salaires entre les officiers du rang et ceux de la gendarmerie étaient flagrants. À cela il fallait ajouter également les écarts importants entre les salaires des officiers étrangers et ceux des officiers ottomans.

Ahmed Niyazi exprime ce malaise dans ses « mémoires » :

«[Devant l'emprise des officiers et agents étrangers sur les affaires de sécurité et des finances], le rôle du pacha inspecteur général (Hüseyin Hilmi Pacha) n'était autre que de satisfaire le désir du palais et des étrangers, il le faisait au-delà des capacités humaines. Après l'insurrection générale et violente et de 1319 (1903), la réponse du gouvernement ottoman n'a pas tardé. Mais cette insurrection fut une belle leçon pour les Bulgares qui avaient atteint leur objectif politique et moral. De toute façon, ils avaient compris qu'ils ne pouvaient pas faire plus. Après cela, on a procédé à la réforme de la gendarmerie et on a même obtenu de bons résultats. Mais en réalité, ces résultats n'étaient rien d'autre que de beaux uniformes et de bons salaires payés régulièrement. Ne parlons pas de lutter efficacement contre les bandits, la réforme n'a même pas rempli son rôle de collecte d'information et d'enquête. Pour cela elle a eu toujours besoin de l'aide de l'armée, de sa protection... Toutes les poursuites, toutes les arrestations se faisaient par les officiers de rang inférieur qui ne recevaient même pas leurs salaires... ${ }^{3}$ "

8 Ainsi, il semble que le rôle de l'armée fut plus qu'un simple passage à l'acte pour devenir le bras armé des Jeunes-Turcs en exil. C'est la raison pour laquelle, en 1906, Ahmed Riza Bey (1859-1930), le leader exilé du mouvement révolutionnaire, fit paraître au Caire plusieurs brochures et notamment le fascicule Devoir et responsabilité : le soldat ${ }^{4}$ où il expliquait le rôle que l'armée était appelée à jouer dans la défense et les progrès de l'Empire ottoman. Ce rôle avait changé, il était passé de la conquête à la défense du pays, de la gaza, au patriotisme. Les officiers étant les éléments les plus qualifiés et les plus patriotes de la nation, il leur revenait de guider la vie politique du pays. En particulier, face au despotisme hamidien qui conduisait l'empire à sa perte, Ahmed Riza demandait à l'élite militaire d'assumer son devoir révolutionnaire, contre le sultan qui, selon lui, avait un seul objectif, celui de se protéger et de protéger ses proches « au prix du sang des soldats ». 
Ahmed Riza Bey

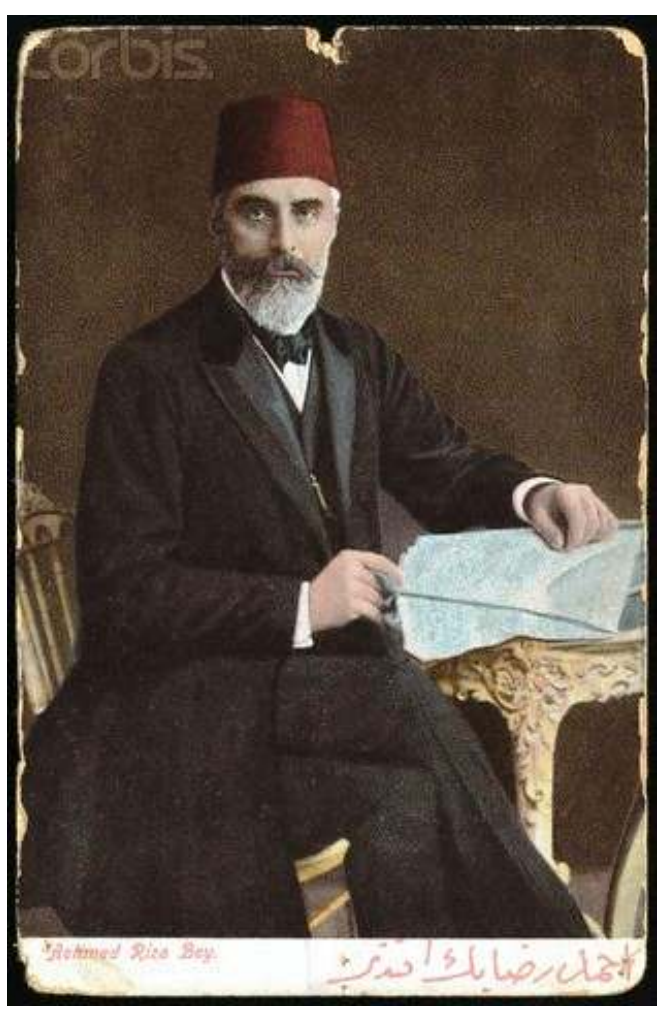

9 Tout en stigmatisant une partie du haut commandement « qui ne vise qu'à s'enrichir, à courir les grades et honneur au mépris des petits officiers et des soldats qui eux courent d'un front à un autre sans même qu'on se préoccupe de savoir s'ils sont capables de supporter le climat, s'ils ont à boire ou à manger, s'ils ont le moindre médicament». Ahmed Riza dénonce dans cette brochure l'utilisation massive de l'élément turc dans les guerres et conflits destructeurs, au bénéfice des autres ethnies musulmanes (albanaise, kurde et arabe), mais aussi des non-musulmans, et demande la généralisation du service militaire à toutes les communautés de l'empire. Mais pour cela le pouvoir doit gagner la confiance de l'ensemble des populations, les utiliser selon leurs besoins et leurs compétences et surtout les nourrir et les équiper. Or, tous ces problèmes ne peuvent être résolus que si l'ordre et la discipline règnent, si l'État a suffisamment de revenus, et si l'utilisation de ces revenus est contrôlée par la « chourâ-i ummet » (l'assemblée de la communauté musulmane). En conséquence,

"chaque soldat a le devoir de demander la réunion du Sénat et de la chambre des députés... Jusqu'à ce qu'un gouvernement constitutionnel qui permette d'unir les différentes communautés musulmanes et de gagner le cour des non-musulmans par la liberté qu'elle accordera soit mis en place, il n'est pas permis de se reposer, car l'ennemi ne s'arrête pas, il profite de notre silence et de notre passivité, pour corrompre le peuple ignorant ».

10 Car, avant le sultan, le soldat doit aimer sa patrie, obéir à la loi et Ahmed Riza invite les généraux écoutés, à l'intérieur comme à l'extérieur du pays, à se réunir et à envoyer des pétitions au sultan et, si nécessaire, à jeter leur épée.

11 C'est dans cet esprit qu'Ahmed Niyazi est formé. Né en 1873, d'un notable albanais, Abdullah Aga, à Resne (Resen), ville située sur la rive nord du lac Prespa à la jonction actuelle de la Macédoine, de la Grèce et de l'Albanie et entourée par les municipalités macédoniennes d'Ohrid, de Demir Hisar et de Bitola (Monastir), "Héros de la Révolution ", le «Garibaldi turc ", Ahmed Niyazi a fait ses études au collège et lycée 
militaires de Monastir. Parmi ses professeurs, nous connaissons Mehmed Tahir de Bursa, auteur du célèbre ouvrage Osmanl Müellifleri (Les Auteurs ottomans). Ses professeurs de français et d'histoire, respectivement les capitaines Orhan et Tevfik lui inculquent «la loyauté, l'humanisme, le patriotisme, le progressisme» par le biais de l'histoire ottomane et de l'histoire de France. Dans les mêmes établissements, il est très influencé par les écrits de Namık Kemal et, notamment, par le célèbre poème de cet auteur, «Liberté ». Ce distique qu'il cite semble l'avoir marqué pour toute son action militaire et politique :

Hakîr olduysa millet, şânnna noksan gelir sanma

Yere düşmekle cevher sakıt olmaz kadr ü kıymetden

Ne pense pas que la gloire du peuple diminue avec l'humiliation

Le joyau ne perd pas de sa valeur en tombant par terre.

Lors de sa formation à l'École de guerre à Istanbul, il a adhéré à l'association clandestine Union et Progrès. Puis, en tant que lieutenant (mülâzım), en 1896, il intègre l'armée où il observe toute sorte d'abus et d'injustices commis par sa hiérarchie. Il participe à la guerre gréco-turque de 1897, déclenchée à la suite de l'insurrection en Crète, où il combat héroïquement dans les batailles de Beşpınar en Thessalie, de sorte qu'il « confirme auprès de l'opinion publique le courage des officiers sortis des écoles militaires ». Pour lui, cet acte héroïque était destiné également à prouver au sultan la qualité de ces écoles et des officiers qui en sortaient. Après la guerre, il se rend à Istanbul avec « ses » prisonniers de guerre. Malgré les propositions du sultan, il refuse d'entrer au palais comme aide de camp et préfère rentrer dans son pays. Cette décision est motivée par la campagne lancée par le sultan contre les Jeunes-Turcs en général et surtout contre ceux qui étaient en exil. C'est à cette époque aussi que Murad Bey (Mizanci), l'une des figures marquantes de l'opposition en exil en France, rentra à Istanbul, en renonçant à ses activités, à la suite de négociations menées avec les représentants du sultan et moyennant l'octroi d'un poste. Ce fut la grande déception de tous les jeunes idéalistes. Niyazi Bey exprime cette immense déception en ces termes :

\footnotetext{
"À cette époque, avec le retour de Murad Bey, l'opinion publique perdit tout espoir, le point d'appui de toute une foule de jeunes, de tous les idéalistes a été ébranlé; certaines personnes au caractère faible changèrent le bonheur et la richesse de l'amour de l'intérêt public et de la sécurité du peuple contre la fortune et l'or offerts par la cour. Cette attitude jeta dans le désespoir toute une jeunesse qui avait accordé sa bienveillance et son respect à ces gens-là ; désormais il fallait accuser d'hypocrisie et de lâcheté tous ceux qui criaient auparavant à tue-tête 'union et fidélité pour le salut de la patrie!' »'.
} 


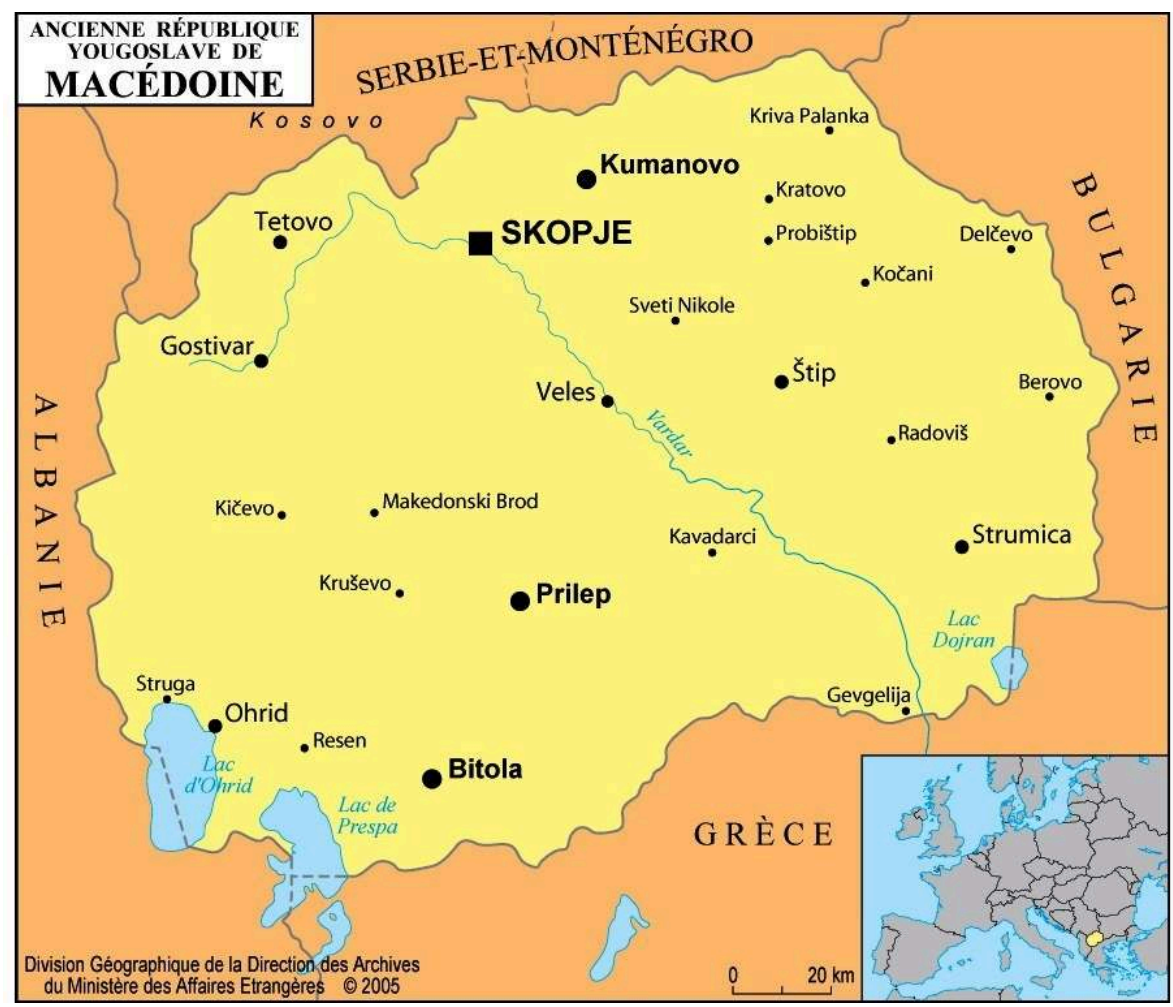

Dépité par le despotisme du sultan, par l'attribution injustifiée des gratifications et des grades aux officiers proches du palais, aux enfants des vieux généraux, Niyazi Bey a regagné Ohrid où il restera, de 1899 à 1903, en tant qu'officier responsable d'un entrepôt de munitions, dans une compagnie de réservistes, autrement dit en service passif. C'est durant ces années qu'il a eu de nombreuses occasions d'observer attentivement à la fois les préparatifs des Bulgares macédoniens pour une révolution et la manière dont les différents missionnaires, instituteurs, officiers et conseillers russes et bulgares contribuaient à l'organisation de cette révolution. Niyazi Bey semble suivre également attentivement les activités des Jeunes-Turcs en Europe et notamment les décisions (et la scission) du Premier congrès à Paris en 1902, par le biais de la presse et surtout par une correspondance avec un certain Mecduddin Efendi, capitaine à Izmir.

Niyazi Bey est tout acquis aux idées d'Ahmed Riza, représentant de la tendance majoritaire et radicale des Jeunes-Turcs, qui s'oppose à toute intervention étrangère et lutte, non pour des réformes ponctuelles locales dans telle ou telle province ou au bénéfice d'une communauté particulière, mais surtout pour une "Révolution totale ». En réalité cette idée de révolution s'exprimait par la revendication du rétablissement de la Constitution de 1876 qui permettrait à chacune des composantes de l'Empire ottoman de jouir des libertés publiques, et pas seulement aux minorités chrétiennes qui " ne demandent que l'intervention des puissances étrangères et le démembrement de l'empire ». Pour ces Jeunes-Turcs les intérêts de la Russie, de l'Autriche et de la cour ottomane étaient les mêmes; toutes les trois visaient à maintenir le statu quo à travers un certain nombre d'aménagements conformément au Traité de Berlin (1878) et notamment la nomination d'un Inspecteur général qui devait être un ottoman musulman, doté des pouvoirs administratifs et militaires et nommé pour trois ans. Très 
mécontents de ces mesures modérées, les comités révolutionnaires macédoniens décident donc de passer à l'action sous la conduite l'ORIM pour une révolution totale.

Créée en 1893, à Salonique, l'Organisation révolutionnaire interne de la Macédoine (ORIM ou VMRO en macédonien), organisait en effet sa révolution, pour le 2 août 1903, le jour d'Ilinden, dans le vilayet de Monastir. Ce fut l'insurrection la plus importante que la Macédoine ait jamais vue jusque-là et ses conséquences furent aussi importantes et sanglantes: plus de 2000 morts, des milliers d'autres blessés et de personnes sans domicile. Les pouvoirs publics ottomans répriment violemment cette révolution avec des troupes qu'ils font venir de l'Anatolie et les responsables sont jugés de manière expéditive par des tribunaux d'exception. Niyazi Bey, mobilisé de nouveau, est affecté dans le troisième régiment de la Troisième armée. C'est durant cette période qu'il joue un rôle actif dans la branche de Monastir de l'Association union et progrès. Fort de sa réputation de "héros de guerre", Niyazi Bey avait visiblement gagné une grande notoriété auprès de la population albanaise musulmane de la Macédoine. Ses promesses d'autonomie pour les Albanais en cas du rétablissement de la Constitution y jouent un rôle déterminant.

Dans la période allant de 1905 à la révolution jeune-turque de 1908 une série d'événements locaux et internationaux met en évidence la dégradation du pouvoir ottoman et inspire de plus en plus confiance aux officiers. Ces événements sont aussi déterminants pour la formation des cadres de l'Association union et progrès et pour la mise en place des structures de cette association. D'abord l'attentat manqué (le 21 juillet 1905) par des Arméniens contre la personne du sultan à Istanbul, l'année suivante, l'assassinat par des Kurdes du maire d'Istanbul, Ridvan Pacha, un proche du sultan; ensuite, dans les années 1906-1907, l'agitation et les révoltes au sein de la population qui refuse de supporter des impôts de plus en plus lourds secouent tout l'empire, comme les mutineries dans l'armée, la troupe ne percevant pas sa solde. Sur le plan international, en 1905, la défaite de la Russie contre le Japon, la révolution qui s'en est suivi et la révolution iranienne de 1906 qui dote le pays d'un régime constitutionnel, sont également de nature à inquiéter le pouvoir et encourager les opposants.

De toute évidence, la création de l'Association ottomane de Liberté, en 1906, à Salonique, correspond bien à cette idée concrète de prise de pouvoir en relation directe avec l'armée. Les premiers membres de l'Association salonicienne étaient essentiellement des officiers et des petits fonctionnaires comme Talat Bey, Ismail Canbolat et Omer Naci. Or, ces hommes étaient guidés, non pas par des théories philosophiques, mais surtout par des conditions sociales et politiques touchant directement le devenir de la Macédoine ottomane, des conditions matérielles de l'armée et des fonctionnaires. La remise en vigueur de la constitution de 1876 était donc pour eux le moyen le plus sûr pour éviter un partage de la Macédoine entre différents États émergents.

En septembre 1907, la fusion dans une seule organisation du groupe de Paris des Jeunes-Turcs dirigé par Ahmed Riza et de l'Association ottomane de Liberté de Salonique, puis la tenue à Paris du second congrès du Comité union et progrès avec le comité arménien Dashnaktsoutioun et la Société pour l'initiative privée et la décentralisation (Teşebbüs-i şahsî ve Adem-i Merkeziyet Cemiyeti) du prince Sabahaddin sont des développements importants pour forcer le sultan, si ce n'est à abdiquer, du moins à rétablir la constitution. Mais la rencontre de Reval (Tallinn), les 9 et 10 juin 1908, entre Edward VII et Nicolas ${ }^{16}{ }^{6}$ semblait être le symbole d'une ingérence 
humiliante et insupportable des grandes puissances pour protéger non seulement leurs propres intérêts, mais aussi ceux des communautés chrétiennes, sans tenir compte des populations musulmanes dans cette région. Une révolution constitutionnelle, comme en Russie ou en Iran, pourrait arrêter le terrorisme, mais aussi les appétits impérialistes. La réponse immédiate à Reval et au silence du sultan est donnée le 11 juin par le Comité union et progrès avec l'attentat contre le commandant de la garnison de Salonique Nâzım Bey, considéré comme l'un des plus grands espions du sultan, qui rapportait systématiquement les activités des Jeunes-Turcs au sein de l'armée et de la population.

19 Après la publication de la longue déclaration du bureau de Monastir de l'Association union et progrès pour expliquer que la question macédonienne n'était qu'un aspect de la défaillance du régime obscurantiste impérial ottoman et pour dénoncer l'attitude hypocrite des puissances européennes, Ahmed Niyazi ne tenait plus en place :

"Je n'étais plus capable de me maitriser; ma volonté était prisonnière d'un sentiment général [...] sous le choc des décisions prises lors de la rencontre de Reval, je me suis débattu durant trois jours et trois nuits dans l'angoisse et inquiétude. Je ne voyais plus aucune autre solution que de mourir. Mais finalement, dans un rêve j'ai vu devant moi dans l'obscurité générale une lumière d'espoir. J'avais trouvé une solution pour atteindre le but " " «J'ai vu que la patrie sous l'ombre de laquelle j'ai grandi, était conduite vers une catastrophe, un grand danger, de manière à ce qu'elle ne retrouve plus la vie et le salut $t^{8}[. .$.$] C'est le moment$ ou jamais pour se révolter contre le gouvernement dont personne n'est content sans distinction de sexe, de classe et religion ${ }^{9}$ ".

\section{Le héros de la Liberté : Hürriyet ya da ölüm, Sloboda ili smrt}

20 Ainsi Niyazi Bey décide "sans hésitation » de créer un çete (groupe armé clandestin) sans attendre un secours quelconque et tient sa première réunion, le 15 juin 1324/28 juin 1908, à son domicile, en compagnie de Cemaleddin Efendi, maire et Tahir Efendi, commissaire de police de Resne ${ }^{10}$, il renouvelle cette expérience le 29 juin avec un groupe plus important ${ }^{11}$, tout en inscrivant sur son kalpak Hürriyet ya da ölüm (la liberté ou la mort), de la même manière que les komitadjis bulgaro-macédoniens qui, eux utilisaient le slogan Sloboda ili smrt (la liberté ou la mort).

21 Finalement, après avoir demandé et obtenu l'accord du Comité union et progrès de Monastir, le 3 juillet, il prend le maquis dans la montagne d'Ohrid en compagnie de 200 hommes et de sa fameuse biche, tout en envoyant un télégramme extrêmement modéré et plein de compassion pour le sultan au palais, à l'inspection générale de Roumélie et au gouverneur de Monastir :

"L'opinion publique veut le rétablissement de la Constitution. La répression exercée n'a pas fait peur au peuple. Au contraire, elle l'a incité davantage et encouragé. De toute façon, le peuple est obéissant à Sa Majesté le sultan et il ne lui demande pas de rendre des comptes pour les maux commis jusqu'à maintenant. Le but essentiel est d'instaurer désormais un régime semblable à celui des pays civilisés, un régime capable de protéger notre pays $d u$ démembrement qui a commencé depuis trente ans et d'établir notre avenir compromis par des nuages sombres sur un texte aux bases solides, et de lui enlever toute idée de pessimisme. Ainsi, alors que chacun doit travailler sans broncher et sans rechigner pour le pays, certains agents secrets ont rempli Salonique afin de commettre des actes susceptibles de créer le désordre. Étant donné que fermer les yeux devant leurs viles actions signifie pour nos compatriotes comme pour les étrangers, les tolérer, le peuple a pris lui-même les mesures nécessaires. De ce fait, armés de fusils Mauser, aujourd'hui, deux cents volontaires ont 
commencé l'action. Pour le moment, plusieurs groupes composés d'ethnies différentes agissent sous le commandement de trois officiers. Notre but, c'est de corriger les traitres et agents qui salissent l'armée et les citoyens patriotes. Si les quatre ou cinq pachas, agents secrets et ceux qui sont nommés avec eux sur une liste spéciale ne repartent pas dans les trois jours qui viennent par un train spécial, tous les gens honnêtes participeront à notre mouvement. Dès aujourd'hui, nous voulons le rétablissement de la Constitution. Si le gouvernement ne le fait pas lui-même, le peuple le fera par la force. En conséquence, notre organisation est fondée pour réaliser ce principe et obtenir la liberté. On verra tout cela très bientôt. Pour le moment, nous ne jugeons pas conforme aux intérêts du peuple et du pays de provoquer d'évènements tragiques, mais l'Association est très décidée à faire partir les personnes corrompues et à protéger l'autorité et l'honneur de Sa Majesté. Pour cela nous voulons la réunion immédiate de la chambre des députés. Sinon la responsabilité appartient au souverain ${ }^{12} . »$

Le bataillon de Niyazi Bey et sa biche

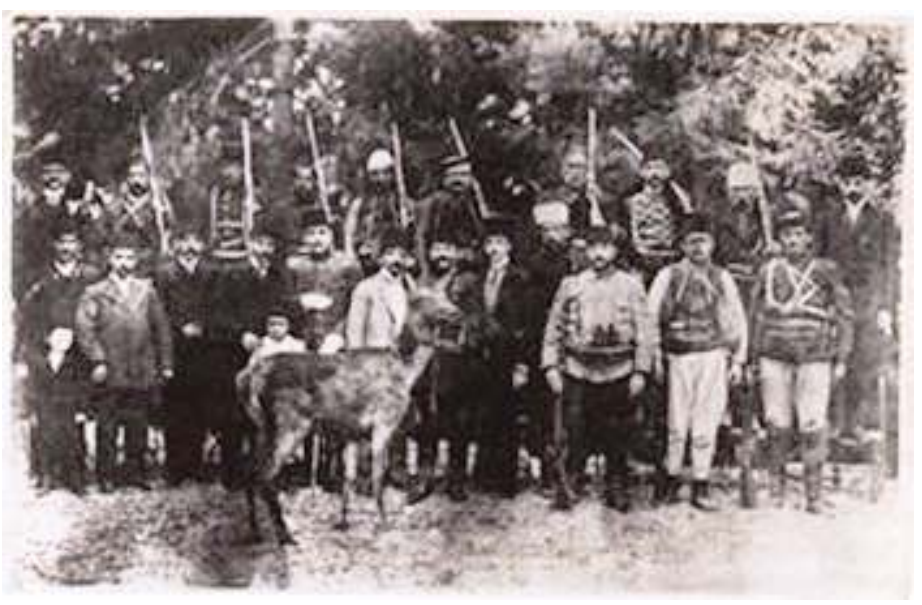

22 À l'exemple de Niyazi Bey, d'autres officiers comme Enver Bey et Eyub Sabri vont aussi faire défection après avoir pillé les munitions dans les casernes. Le sultan répond à ces mutineries par la mutation des responsables de haut rang en nommant Ibrahim Pacha, commandant de la région de Serrès à la place du commandant en chef de la Troisième armée, Esad Pacha. Le sultan mobilise également les réservistes de la Première classe d'Anatolie, il somme par ailleurs şemsi Pacha, commandant de la $18^{\mathrm{e}}$ division à Mitrovitza, un officier albanais des plus fidèles, de se rendre à Ohrid. Arrivé à Monastir, le 7 juillet, il sera abattu par plusieurs balles au milieu de ses hommes par un membre $\mathrm{du}$ Comité union et progrès ${ }^{13}$. Dès ce jour-là, les volontaires albanais qui accompagnaient le pacha sont renvoyés chez eux. Pour les révolutionnaires, cet assassinat est un acte d'encouragement formidable pour la poursuite du mouvement, alors que pour le sultan, il s'agit d'un acte d'impuissance. 


\section{Enver Bey}

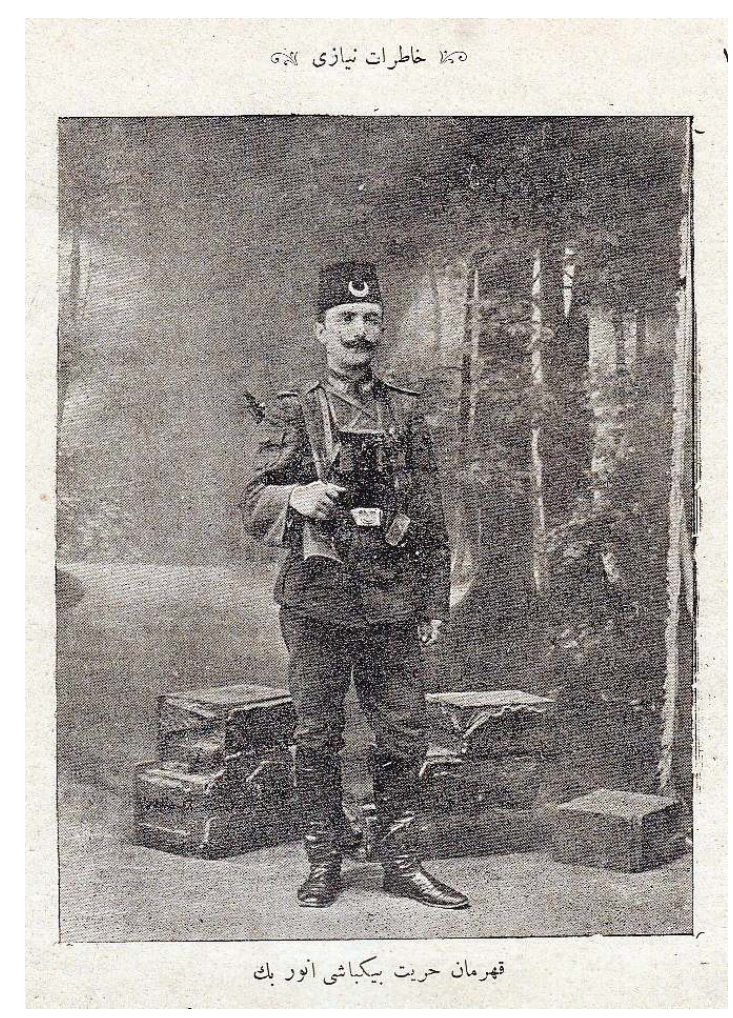

\section{Şemsi Pacha}

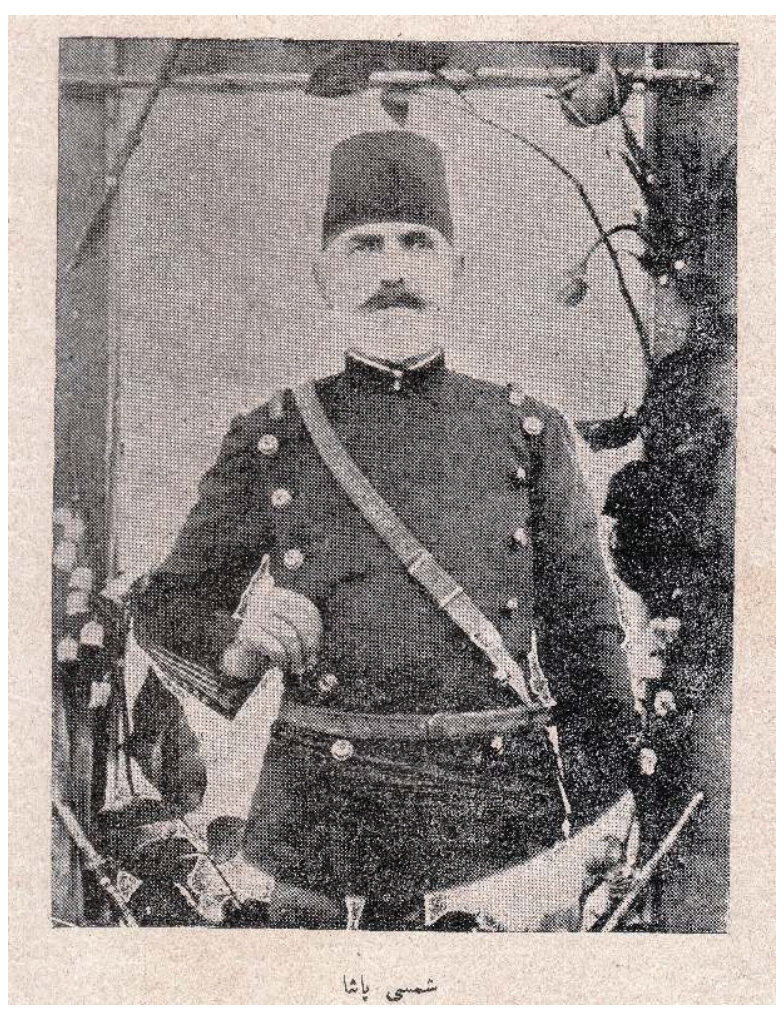

23 Phénomène plus grave, la plupart des représentants du pouvoir dans la région commençaient sérieusement à douter de l'autorité du sultan et les troupes d'Anatolie 
ont refusé de tirer sur leurs camarades. Finalement, le 23 juillet, alors que Niyazi Bey, après plusieurs semaines de maquis, rentrait à Resne, la Constitution est proclamée devant la caserne de Monastir en présence des autorités militaires et civiles de la province. Au même moment plusieurs localités de Macédoine suivent l'exemple de Monastir.

\section{Manifestation à Monastir}

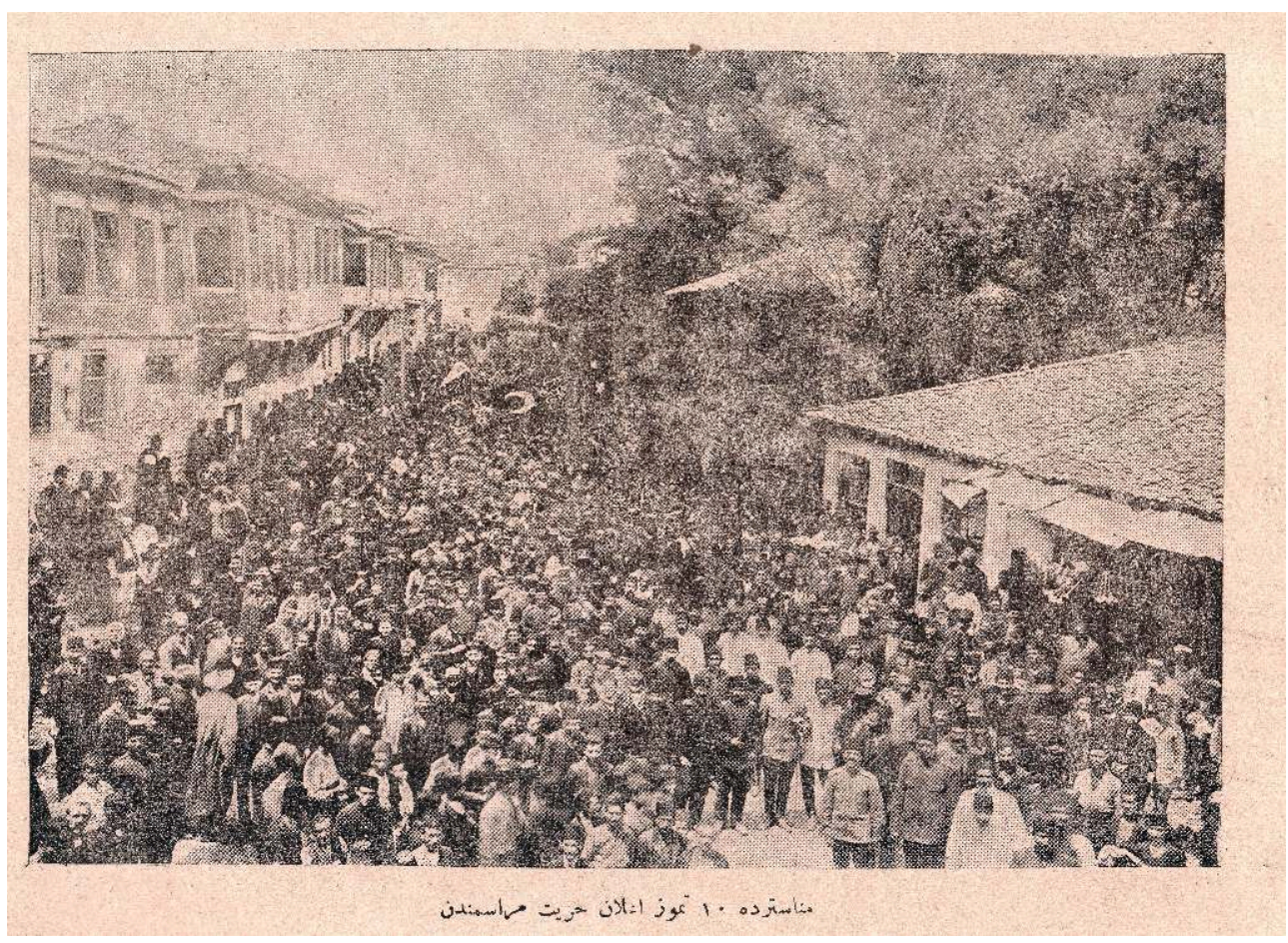

Niyazi Bey fait le récit de la journée du 23 juillet (10 juillet en calendrier vieux style) de cette manière :

"Le jeudi vers midi, alors que nous rentrions à Kichrani, une cérémonie exceptionnelle, accompagnée de tirs de canon se déroulait à Monastir pour la proclamation de la Liberté et toutes les communautés musulmanes et non musulmanes fêtaient dignement cet événement dans une fraternité et une égalité totale. Osman Pacha et moi fument les hôtes de Ferhad Aga. Après avoir déjeuné nous avons poursuivi notre route. Nous sommes entrés à Resne vers le soir. Tous les fonctionnaires civils et militaires, toutes les communautés musulmanes et non-musulmanes et toutes les classes sont venus à notre rencontre, le pacha a reçu un accueil exceptionnel. La maison de Rıza Aga, un notable de Resne, étant consacrée au pacha, nous nous y sommes installés. Ce soir-là, tous les hommes qui m'ont accompagné dans le maquis dès les premiers jours étaient très contents. Chacun rejoignait sa maison, sa famille; la joie et le bonheur avaient envahi tout le monde ${ }^{14}$. 


\section{Manifestation pour la Constitution}

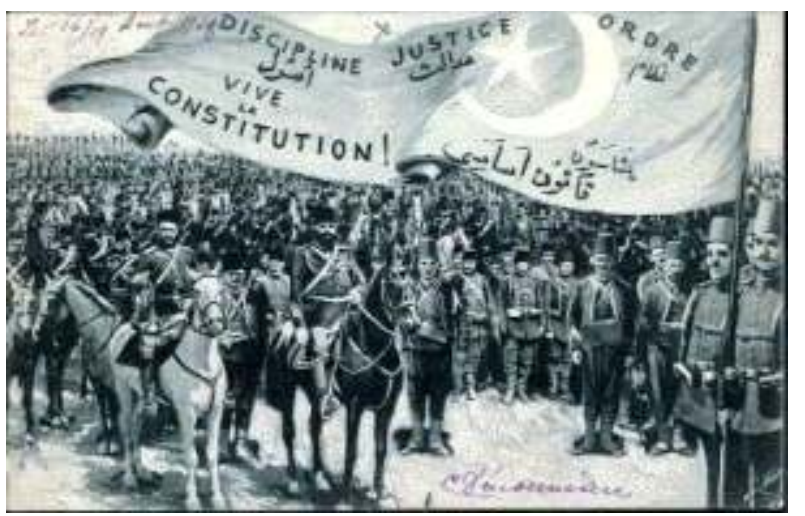

Ainsi, les villes ont été investies par la foule, tous les comités bulgares, serbes, grecs, turcs sont descendus de la montagne, les rues étaient remplies d'affiches dans toutes les langues parlées en Macédoine scandant la Constitution : "Yaşasın Kanûn-i Esâsi »,

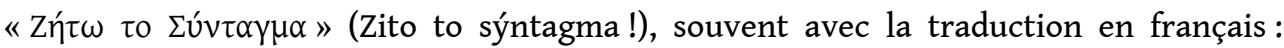
«Vive la constitution! ». Des cartes postales aux effigies de Niyazi Bey, « héros de la Révolution » et d'Enver Bey (puis pacha) sont imprimées rapidement.

Abdülhamid II entre Enver Bey et Niyazi Bey

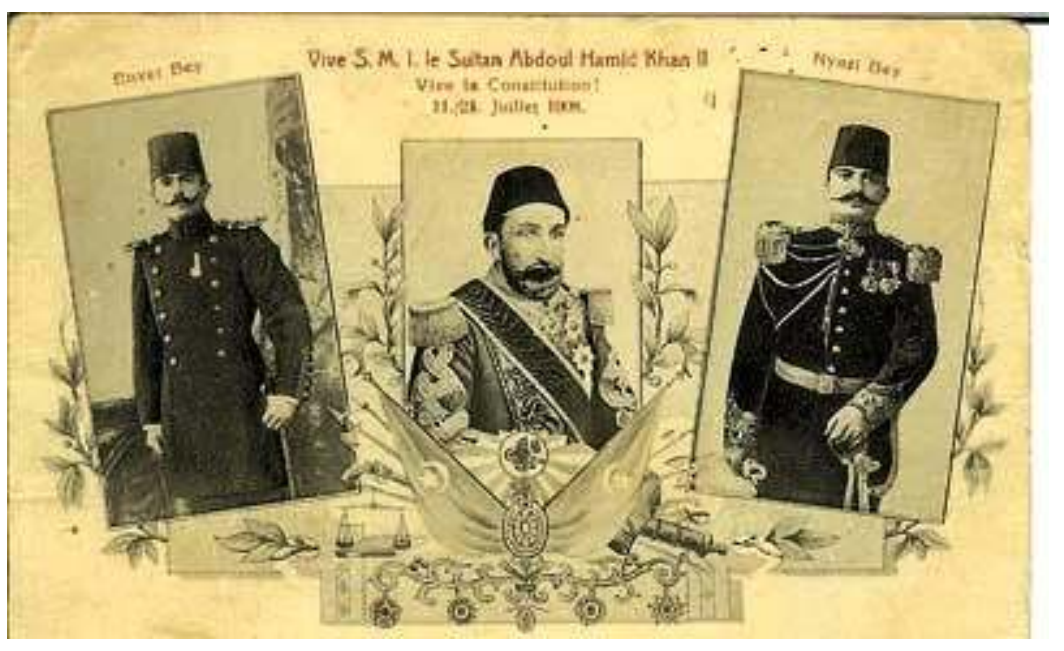

L'affiche la plus importante, une imitation de " La Liberté guidant le peuple ", d'Eugène Delacroix est tout un programme : en toile de fond, la ville d'Istanbul surmontée des armes impériales, au centre Namik Kemal (1840-1888), accompagné de trois pachas, console «la Liberté », représentée par une femme orientale, élégamment vêtue et fraichement débarrassée de ses chaînes par un Niyazi Bey aux grosses moustaches albanaises et Enver Bey (celui qui tient la masse) ; au fond, à gauche, "le peuple » maintenant uni et mêlé en congratulations et embrassades réunit toutes sortes d'ethnies composant l'Empire ottoman. On y distingue les drapeaux turcs, arabes, grecs, arméniens, etc. Le tout est protégé par un ange descendant du ciel avec un fanon sur lequel sont inscrits les slogans de la Révolution française, en plus du mot «justice »: «Hürriyet, Adalet, Musavat, Uhuvvet (Liberté, justice, égalité, fraternité). Enfin tout à droite en haut est inscrit : « Devlet-i Osmaniye'nin ihyast» (la renaissance de l'État ottoman) et tout à gauche en bas est inscrit en turc ottoman et en grec "Yaşasin

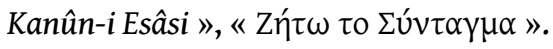


«La liberté guidant le peuple »



Cette liesse est naturellement amplifiée par des chansons et marches dont la plus importante commence par « Yaşasın Niyazi'ler, Enver'ler... » (vive les Niyazi, les Enver...). Mais voici une autre, tardive celle-là, datant probablement des 1910, lorsque les JeunesTurcs avaient renoncé à l'ottomanité pour une turcité idéologique :

Milletin fahri Niyazi, ordumun Enverleri

Muhterem cemiyetin ey kahraman askerleri

Azm-ı kati samı ceri ittihat rehberleri

Devleti ihya eden şura-yı ümmet erleri

Ordumuz etti yemin

Titredi hak u zemin

Milleti etti emin

Açıldı rah-ı nevin

Sancağımız şanımız

Türk oğludur sanımız

Vatan bizim canımiz

Feda olsun kanimiz. ${ }^{15}$

La gloire du peuple, Niyazi, les Enver de mon armée

ô les soldats héroïques de l'Association respectable

Les Guides de l'Union à la volonté ferme et à l'oreille bien sensible

Les soldats qui ont ressuscité l'État de la communauté

Notre armée a prêté serment

La terre et le ciel ont tremblé

Elle a mis en sécurité le peuple

Une voie lumineuse est ouverte

Notre honneur, c'est notre drapeau

Notre surnom est fils de Turc

La patrie est notre âme

Que notre sang y soit sacrifié 

l'Association union et progrès, les « Mémoires » de Niyazi Bey constituent certainement une des meilleures sources pour sa biographie de la période allant de 1887 à 1908 , relatant surtout son action militaire et politique en Macédoine. Ces mémoires sont aussi pleines de contradiction. Visiblement, la notoriété personnelle qu'il a gagnée au cours de la Révolution lui a été quelque peu reprochée par sa hiérarchie militaire et politique et il se sent dans l'obligation de se justifier dès le départ en disant :

"c'est pour exprimer et prouver que ce que j'ai fait ne vaut pas ces applaudissements et ces éloges que j'ai été obligé de rédiger ces mémoires. Qu'ai-je fait? Je ne le sais. Si, le destin qui m'a désigné pour me trouver à Resne au bon moment, l'avait fait pour un autre camarade, celui-ci aurait-il fait moins que moi ?...Le verdict populaire qui blesse profondément la conscience collective à mon égard est une faute grave... C'est une calomnie ! ${ }^{16}$. et progrès qui, selon lui, représente le peuple, et lui-même a désormais avec les JeunesTurcs le régime qu'il mérite. C'est à la fois vrai, car ces décisions étaient collectives et anonymes, mais c'est aussi une manière de montrer qu'il était gêné par ces manifestations en sa faveur, manifestations qui faisaient ombrage aux cadres supérieurs comme Enver Pacha. Niyazi prévient le lecteur dans l'introduction qu'il ne doit pas attendre de révélations sur l'histoire et la manière dont l'Association fut créée ni sur les fondateurs ${ }^{17}$. C'est pour éviter aussi des égarements possibles dans ses mémoires qu'il dit ne pas les avoir rédigées complètement.

Cela dit, au cours de son texte et à travers les documents qu'il publie, et notamment les télégraphes et lettres qu'il a envoyés ou qu'il a reçus, il ne cesse d'insister sur l'action 
qu'il a menée personnellement et avec son groupe. Il y a bien une photo d'Enver Pacha dans ses mémoires portant la mention «Le héros de la Liberté, le capitaine Enver Bey $»^{18}$, mais il n'en parle pratiquement pas. En revanche, les mémoires de Niyazi Bey regorgent d'informations sur la région du lac de Prespa, les villes d'Ohrid et de Resne et surtout sur les personnalités albanaises ayant pris part à la Révolution jeune-turque. Il serait extrêmement utile de les traduire en français et de les publier.

Halil Pacha (Kut), (1972), Ittihat ve Terakki'den Cumhuriyet'e Bitmeyen Savaş [La guerre ininterrompue depuis l'Union et progrès jusqu'à la République], Istanbul.

\section{BIBLIOGRAPHIE}

Bilici, Faruk, (2007), « La Macédoine ottomane et Necati Cumalı », dans Necati Cumalı, Macédoine 1900, traduction, introduction, postface et notes, Arles : Actes Sud-Sindbad, collection «Bibliothèque turque », p. 253-281.

Bilici, Faruk, (2006), « Les Jeunes-Turcs et la Macédoine (1908-1912) : la province qui fit vaciller l'Empire », Mustafa Kaçar, Zeynep Durukal (dir.), Essays in Honnor of Ekmeleddin Ihsanoğlu. Societies, Cultures, Sciences: A Collection of Articles, vol. I, Istanbul: Research Centre for Islamic History, Art and Culture (IRCICA), p. 87-102.

Georgeon, François, (2003), Abdulhamid II, le sultan calife, Paris : Fayard, 2003.

Niyazi, Ahmed Resneli, Hâtırât-ı Niyâzi yahut Tarihçe-i Inkilab-ı Kebîr-i Osmaniden bir Sahife [Mémoires de Niyazi ou Une page de l'histoire de la Grande révolution ottomane], Istanbul.

\section{NOTES}

1. Faruk Bilici, «La Macédoine ottomane et Necati Cumalı », dans Necati Cumalı, Macédoine 1900, traduction, introduction, postface et notes, Arles, Actes Sud-Sindbad, collection «Bibliothèque turque », 2007, pp. 253-281; Faruk Bilici, «Les Jeunes-Turcs et la Macédoine (1908-1912) : la province qui fit vaciller l'Empire ", Mustafa Kaçar, Zeynep Durukal (dir.), Essays in Honnor of Ekmeleddin Ihsanoğlu. Societies, Cultures, Sciences: A Collection of Articles, vol. I, Istanbul : Research Centre for Islamic History, Art and Culture (IRCICA), 2006, pp.87-102.

2. Halil Pacha (Kut), Ittihat ve Terakki'den Cumhuriyet'e Bitmeyen Savaş (La guerre ininterrompue depuis l'Union et progrès jusqu'à la République), Istanbul, 1972, pp. 30-31.

3. Resneli Ahmed Niyazi, Hâtırât-ı Niyâzi yahut Tarihçe-i Inkilab-ı Kebîr-i Osmaniden bir Sahife (Mémoires de Niyazi ou Une page de l'histoire de la Grande révolution ottomane) (infra: Ahmed Niyazi, Hâtırât) pp. 32-34.

4. Fascicule II édité en Égypte, 1323 (1906), 69 p.

5. Ahmed Niyazi, Hâtırât, pp. 20-21.

6. Le 9 et 10 juin 1908 a eu lieu à Reval un sommet entre le tsar Nicolas II et le roi d'Angleterre Édouard VII, au cours duquel les traités conclus en 1907 et la convergence de vues sur la situation en Perse, en Afghanistan et en Macédoine ont été réaffirmés.

7. Ahmed Niyazi, Hâtırât, p. 63. 
8. Ahmed Niyazi, Hâtırât, p. 64.

9. Ahmed Niyazi, Hâtırât, p. 67.

10. Ahmed Niyazi, Hâtırât, pp. 63-64.

11. Ahmed Niyazi, Hâttrât, p. 69.

12. Ahmed Niyazi, Hâtırât, p. 83.

13. François Georgeon, Abdulhamid II, le sultan calife, Paris, Fayard, 2003, pp. 397-400.

14. Ahmed Niyazi, Hâtırât, p. 230.

15. http://www.uzunkopru.pol.tr/trhce

16. Ahmed Niyazi, Hâtırât, p. 11.

17. Ahmed Niyazi, Hâtırât, p. 13.

18. Ahmed Niyazi, Hâtırât, p. 16.

\section{RÉSUMÉS}

Si la Révolution jeune-turque de 1908 a été préparée et alimentée par des élites dans les grandes villes ottomanes, sans l'implication directe de l'armée, elle ne pouvait avoir lieu. Né à Resne (Resen), d'un notable albanais, Abdullah Aga, Ahmed Niyazi (1873-1913) est un héros de la Guerre turco-grecque de 1897, comme de la Révolution jeune-turque, car il fut le premier à prendre le maquis. Par la suite, on chantera ses louanges ainsi que celles d'Enver Pacha, mais lui faisant probablement ombrage, Ahmed Niyazi sera tué par les proches d'Enver Paşa.

Although the elites prepared the Young Turks Revolution of 1908 and provided it strength in the big Ottoman cities, it couldn't have been possible without the direct involvement of the army. Son of Abdullah Ağa, an Albanian notable and born in Resne (Resen), Ahmed Niyazi (1873-1913), who was the first to go underground, is a hero of this Revolution and a hero of the Greco-Turk war of 1897. He was highly praised alongside Enver Paşa who probably took umbrage at him and whose close friends murdered him.

1908 Genç Türk İhtilâli büyük şehirlerde aydınlar tarafından hazırlanıp beslendiyse de Ordunun doğrudan müdahalasi olmadan gerçeklemesi olanaksızdı. Arnavut eşraftan Abdullah Ağa'nın oğlu olarak Resne (Resen)'de doğan Ahmed Niyazi (1873-1913) 1897 Türk-Yunan şavaşı kahramanı, daha sonra ilk önce dağa çıkan kişi olarak İhtilâl kahramanı oldu. Niyazi Bey Enver Paşa'nın yanında anılacak, ancak muhtemelen ona gölge yaptığı için yakınları tarafından öldürüldü. 
INDEX

Index chronologique : Empire ottoman, révolution jeune-turque (1908-1914)

motsclesmk БИТОЛА, ОТОМАНСКАТА ИМПЕРИЈА, ОХРИД, СКОПЈЕ, СОЛУН

Index géographique : Monastir, Ohrid, Resne, Salonique, Skopje

motsclestr Genç Türk İhtilâli (1908-1914), Monastir, Osmanlı İmparatorluğu, Resne, Resne’li

Niyazi Bey Ahmed (1873-1912), Selanik, Üsküb

Thèmes : Histoire

Mots-clés : Niyazi Bey Ahmad (1873-1912), Association ottomane de Liberté, Çete/chette/ tchete/tsete, CUP, Dashnaktsoutioun ou Dachnak, Enver Bey Ismail (1881-1922), Hilmi Pacha (1855-1922), Jeunes-Turcs, Kalpak, Namık Kemal (1840-1888), Niyazi Bey Ahmed (1873-1912), ORIM/VRMO, Reval (rencontre) (Tallinn)

Keywords : Resne, Ottoman empire, Young Turks revolution (1908-1914), Niyazi Bey Ahmad (1873-1912), History

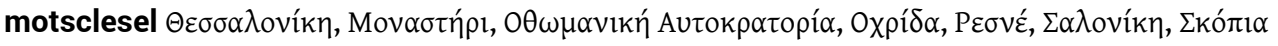

